\title{
STUDIES OF BEAM INDUCED RADIATION FOR EXPERIMENT 735 \\ AT THE CO INTERSECTION REGION \\ AND ITS EFFECT ON DETECTOR COMPONEN'TS
}

F. Turkot and C. Hojvat

Fermilab

W. Anderson and C.S. Lindsey

Iowa State University

N. Biswas and J. Piekarz

University of Notre Dame

A. Bujak

Purdue University

June 12,1985 
Studies of Beam Induced Radiation for Experiment 735

at the CO Intersection Reqion and its Effect on

Detector Components

Frank Turkot and Carlos Hodvat

Fermilab

Walter Anderson and Clark S. Lindsey

Iowa State University

Nripen Biswas and Jadwiga Piekarz

University of Notre Dame

Adam Bujak

Purdue University

June 12th, 1985.

\section{Introduction.}

For collider detectors Iike E-735 there is a general concern about the effects of background radiation as they share the same beam enclosure as the accelerator. In the case of the Fermilab collider, the cycles for production of antiprotons with the Main Ring may overlap a significant fraction of a collider data-taking run. During this time 10 to 20 times as many protons will be injected, accelerated and extracted from the Main Ring as compared to the number present in the collider itself.

In this regard E-735 is at a disadvantage when compared to other detectors to be installed in BO and DO. In the case of BO, a large overpass will completely remove the Main Ring from the detector's enclosure for runs after the first one in the summer of 1985. The Do detector will utilize a presently existing bypass that locates the Main Ring just above the sensitive area of the apparatus. In addition. E-735 shares the tunnel with the abort devices for both the Main Ring and the Tevatron. Although the radiation problems that E-735 will encounter will be shared to some extent by the other two larger detectors, enough differences exist in geometry as to warrant an independent study.

The E-735 collaboration has started a series of measurements in order to evaluate the amount of radiation present in the Collider tunnel under different circumstances and its effects on:

1) Scintillators, phototubes and their electronics.

2) Possible damage to drift chambers wires due to high counting rates.

3) Possible damage to the drift chamber electronics.

4) Evaluate backgrounds and resulting trigger rates.

5) Study the effect of local shielding. 
Beginning in March 1985, radiation measurements were started in the Main Ring tunnel at co during beam-on operation in order to evaluate the implications for the experiment. This note presents out results.

Measurement set-up

The detectors used for these studies include:

1) Two arrays of Lif crystal dosimeters (TLD's), 24 in total.

a) Three scintillation counter telescopes:

(a) $A 1, A 2, A 3, A 4 ; 1 " \times 3$ " counters

(in part of the running 2 " of Pb was placed between $A 1$ and $A 2$, and $4^{\prime \prime}$ between $A 3$ and $A 4$ ).

(b) B1, B2, B3; 0. 4"x0. 55" counters, remote positioning

(c) $\mathrm{C} 1, \mathrm{C2} ; 5.7^{\prime \prime} \times 33^{\prime \prime}$ counters

3) A standard Fermilab Scarecrow ion chamber modified to 1E-4 Rads/pulse and a 1 second time constant.

(for the first part of data taking a 6.25 microrads/pulse and 20 seconds time constant was used).

4) Six sample pieces of Bicron plastic scintillator (types 408, 412, 434), polyvinyltoluene base.

Top and side views of the detector geometry at co, with respect to the Main Ring and Tevatron beam pipes are shown in Figures 1 and 2. A PDP11/45 computer system was set-up in the co service building for data recording. The TLD's are removed every two to three weeks by the Radiation Physics group for analysis, giving only the integrated doses received during that period.

\section{Results}

As the fermilab accelerators presently operate in a variety of modes we have selected preliminary results for three very different ones.

In mode 1 the data correspond to the Tevatron operating at 800 Gev for fixed target physics, with the Main Ring simmultaneously providing protons at $150 \mathrm{GeV}$ (for HEP) and at $120 \mathrm{GeV}$ and $8 \mathrm{GeV}$ (for antiproton source commissioning and/or tune-up).

We present the data as Krads per 1 els protons to obtain numbers that can be related to a collider run. In principle, 1000 hrs of Collider operation with a luminosity life-time of $20 h \mathbf{s}$ will require a minimum of 50 refills of antiproton bunches. Assuming that a refill requires 10 hrs of antiproton production at $2 E 12$ protons every 3 seconds we obtain a total number of protons accelerated per collider run of 1.2E18. 
In Figure 3 we summarize the results obtained with the TLD's vs. position with respect to the Main Ring and Tevatron beam pipes for operation in mode 1 . This data was obtained during operation for fixed target physics. In addition, we show the data obtained with the ion chamber and its relative position to the TLD detectors. The agreement between the two sets of data is quite good. The maximum observed dose varies between $49 \mathrm{Krads}$ for the TLD's to the order of 30 Krads per 1 E18 protons for the ian chamber. The observed integrated dose appears to be dominated by losses in the Main Ring and to be associated with the many parasitic tuning ramps under each Tevatron cycle.

In addition, for a period of 4.5 weeks for this type of operation. the samples of scintillator received doses of $30 \mathrm{krads}$ and 21 Krads. In Figure 4 we present the pulse height obtained with a ruthenium source versus distance along the scintillator for the three types tested comparing the unexposed samples with the exposed ones. The actual length of scintillator required for the experiment is 40". (A detail report on scintillator degradation is in preparation).

Finally, radiation resulting from beam aborts, at co, in both the Main Ring and the Tevatron was examined during mode 1 operation. In a sample of 100 Tevatron aborts at intensities between 0.5 and $1.2 E 13$ circulating protons, we set an upper limit of 15 mRads/abort. Similarly with Main Ring aborts at $150 \mathrm{GeV}$ we have an upper limit of 12 mRads/abort.

In mode $a$ results were obtained during 4 hrs of Main Ring operation dedicated to the Antiproton Source. Ten 120 Gev cycles per minute, at close to $1 E 12$ per cycle, were extracted towards the antiproton production target. This is the only occasion that the Main Ring has operated essentially in the production mode to occur during collider runs. Due to the shortage of the run no TLD measurements were possible, but with the ion chamber we obtain a dose of 1.9 Krads per 1 El8 protons. The spatial distribution can be seen in Figure 5 , as singles in the remote scanning $B$ telescope; the solid lines are the relative TLD data from figure 3 in arbitrary units. The dependence of counting rate with vertical position is steeper than the TLD data for mode 1 , since there is no contribution from the Tevatron beam in this mode. The ion chamber time dependent data indicates that nearly all of the dose occurs in the first 0.5 seconds following injection into the Main Ring (the time resolution being limited by the ion chamber response). Looking at the anode current of the scintillator counters, $80 \%$ of the losses ocurrs in the first $10 \mathrm{~ms}$ after injection, with the rest ocurring between start of acceleration and transition time. Hence in the antiproton production mode essentially all of the losses will be limited to a 0.3 seconds interval following Main Ring injection.

other results of interest were obtained in mode 3 , also during the short antiproton production run. During this period, tuning of the bunch rotation rf system in the Main Ring caused all the 
accelerated beam to be lost within the tunnel, with essentially no beam going either to the abort line or to the antiproton target.

This mode resulted in the largest doses we have seen so far, of the order of $220 \mathrm{Krads}$ per 1 E18 protons.

\section{Conclusions-Radiation Dose}

Based on the measurements and analysis described above we draw the following conclusions about the radiation enviromment at co:

1. - During a "clean" 1000 hrs long collider run

(1E18 protons injected in Main Ring) the maximum integrated dose to any element of the detector will be of the order of 3 Krads. In Figure 6 we show the predicted dose distribution at the detector using the TLD data from mode 1 for shape (Figure 3 ) and the ion chamber data from mode 2 for normalization.

2. - Essentially all of the dose is due to losses in in Main Ring during the first 0 . 3 seconds following injection.

3. - Doses 100 times larger can be generated by tuning and/or machine studies that result in most of the circulating Main Ring beam being lost around the tunnel, instead of going to the abort channel.

4. - Modest amounts ( 3 " lead) of local shielding can reduce dose rates by $50 \%$. We have not yet found a practical way of applying this to the actual apparatus.

\section{Conclusions-Implications for the Detector.}

1. - There will be no significant degradation of the scintillator during a "clean" collider run.

2. - The gas gain in all chambers will be reduced by a factor of 100 during the 0.3 sec of beam loss.

This will result in $10 \%$ dead time for data taking, assuming a 3 sec cycle for antiproton production.

3. - The preamplifier chamber electronics will be remoted by the order of $10^{\prime}$ to an area inside the entrance to the spectrometer room to reduce the dose per run to the order of 300 rads.

4. - In order to cope with potentially high dose rates during aborts (we have so far not observed such losses) or erratic machine pulses, it appears feasible to reduce the cylindrical chamber gas gain in a period of 5 to 10 microsec. We plan to use the trigger for the abort kickers to initiate this process. We plan to study this at co with a small prototype chamber in the next two months.

5. - Gain stability of photamultipliers in the trigger hodoscope- We must limit the average anode current to 
less than $100 \mathrm{mictoA}$ during the 0.3 sec period of potential losses, in order to keep the overall average current below $10 \mathrm{microA}$. We will investigate pulsing down the voltage of a dunode uith our counters existing at $\mathrm{CO}$.

6. - That portion of the detector in the turnel itself must be removed during fixed target operation of the accelerator. 
When the detector (we suspect this conclusion to extend equaliy to other detectors in the collider tunnel) is in place within the tunnel, the accelerator must operate in a relatively clean mode. Regular machine studies should not result frequently in the total loss of circulating beams. Coordination with the Accelerator Division will be required to limit the dose deposited at co (and other experiments locations) to less than 5 to $10 \mathrm{Krads}$. 


\section{References}

"Compilation of Radiation Damage Test Data"

Parts 1 and 2 , Cern 79-04, H. S. Schonbacher et al.

Part 3, Cern 82-10, P. Beynel et al.

"Detector Development", B. Pope,

Proceedings 1984 SSC Summer Study. Snowmass.

"Radiation Damage and Rate Limitations in Tracking Devices", M. G. Gillchriese, ibid. 
$\therefore$
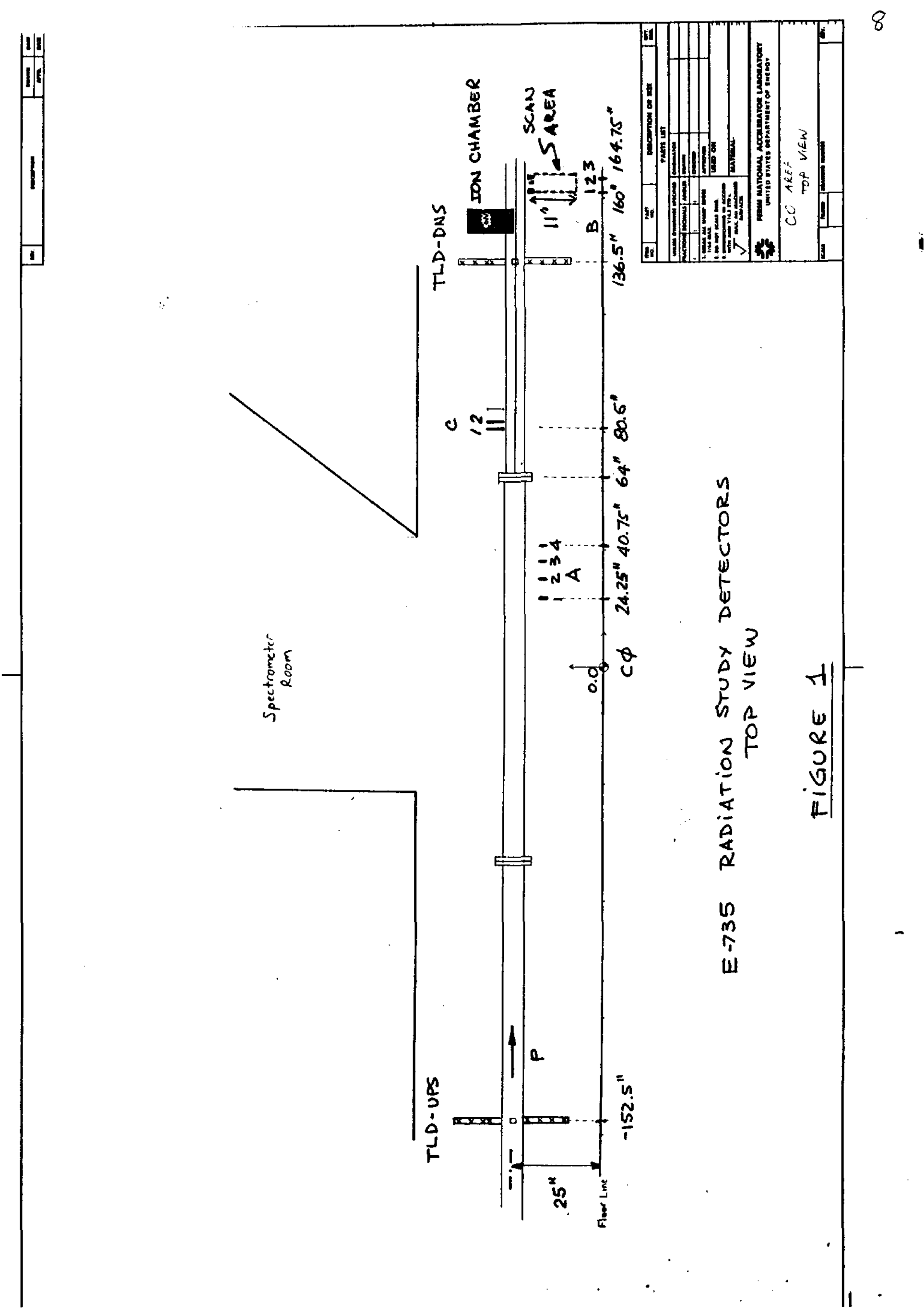


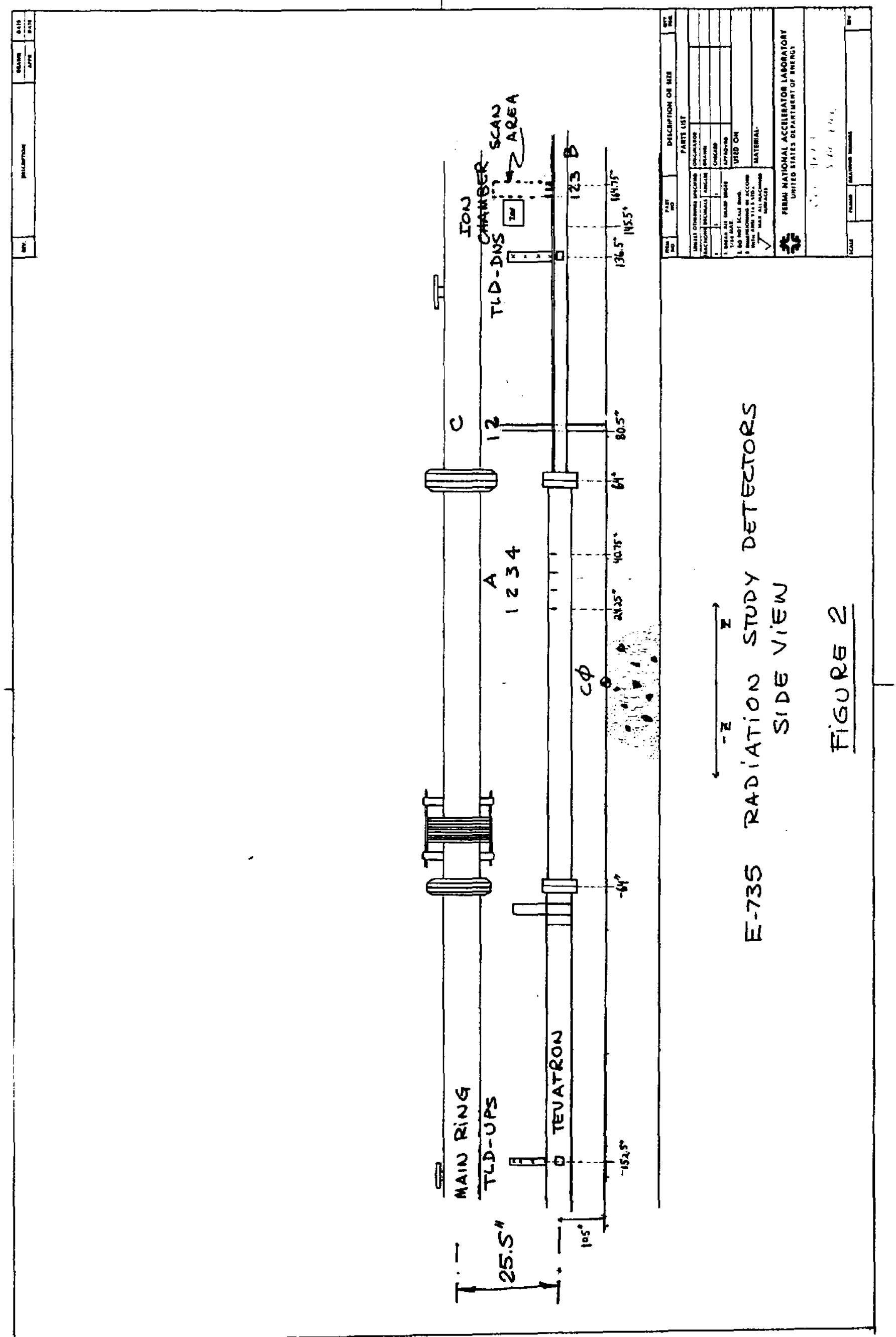




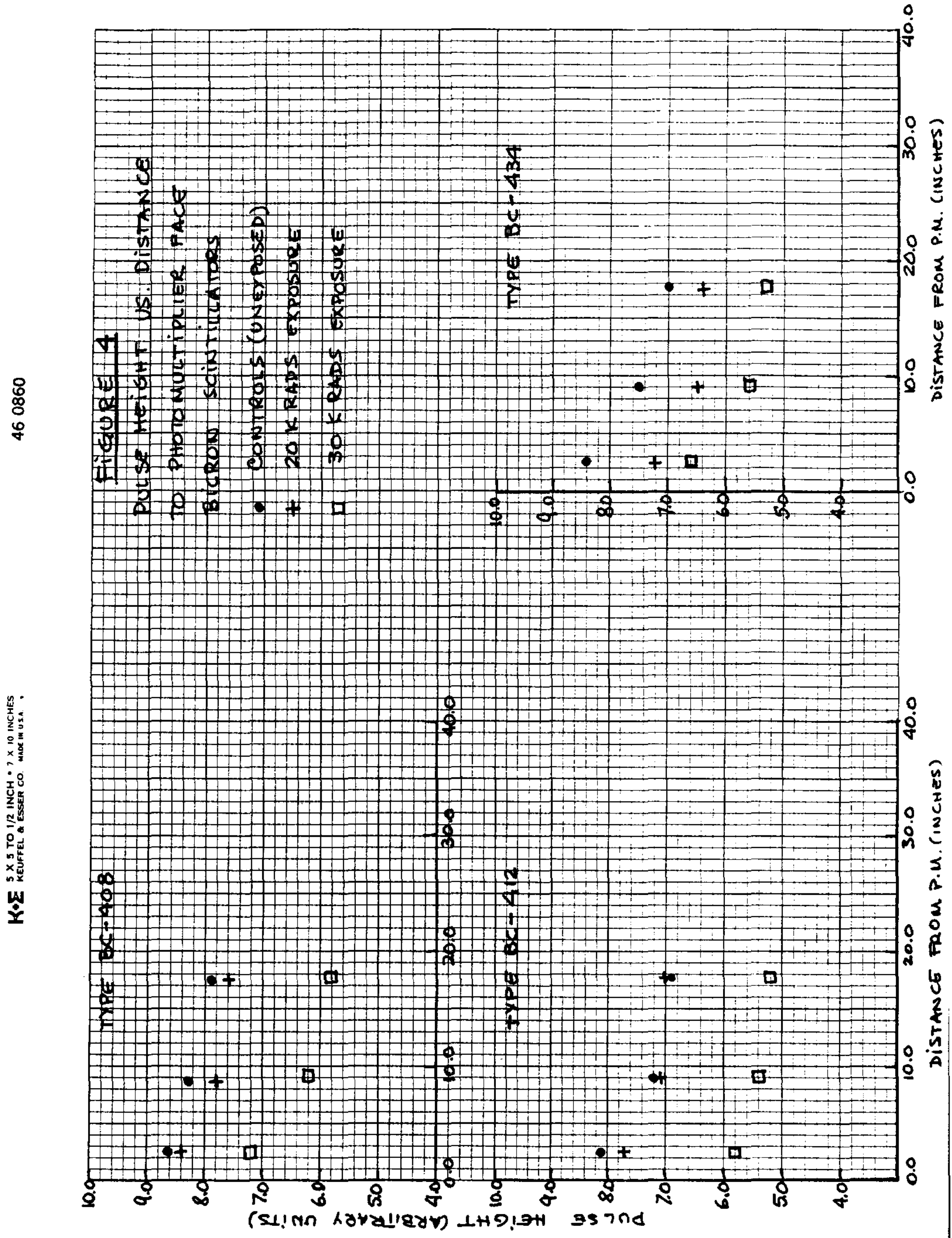




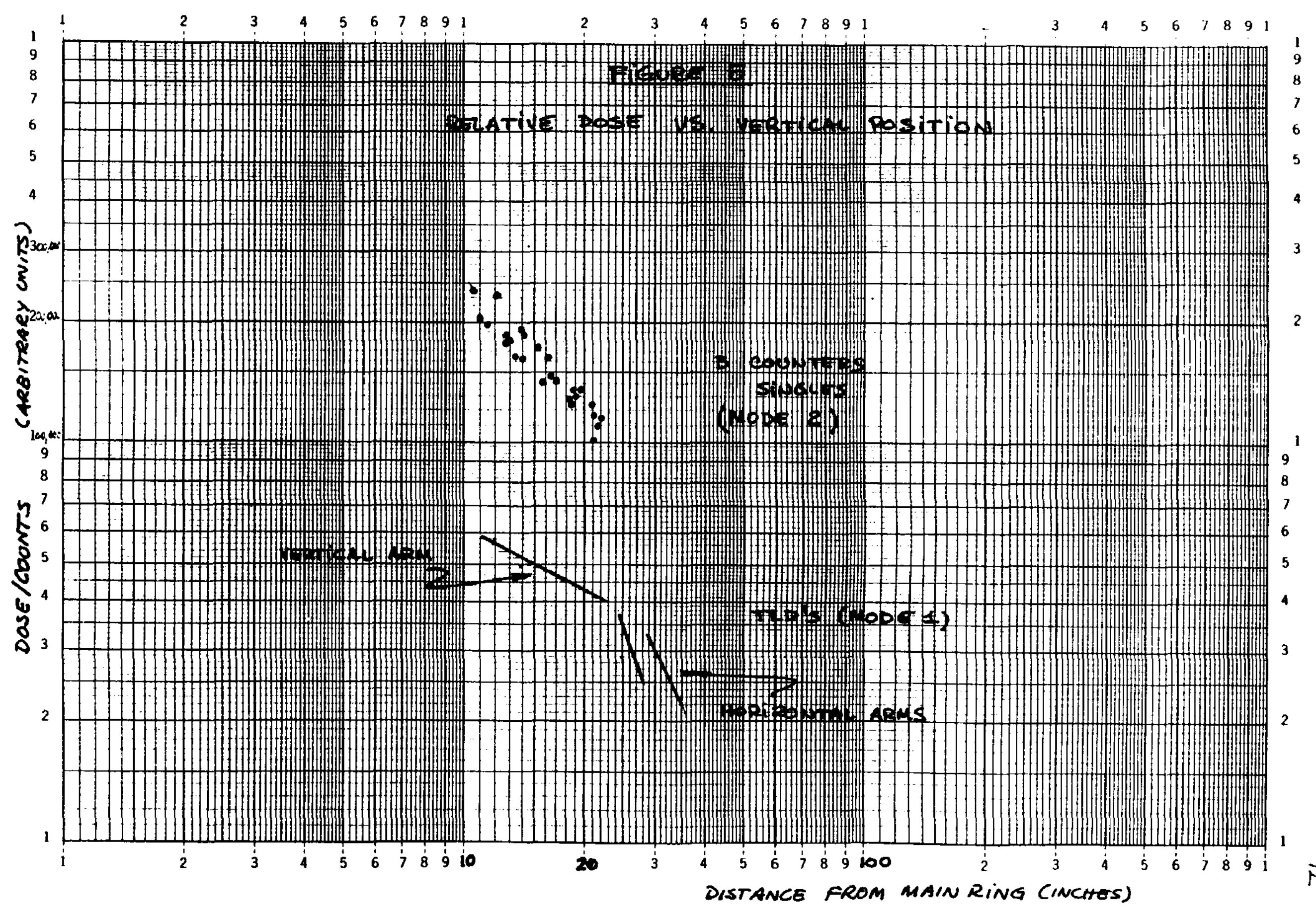




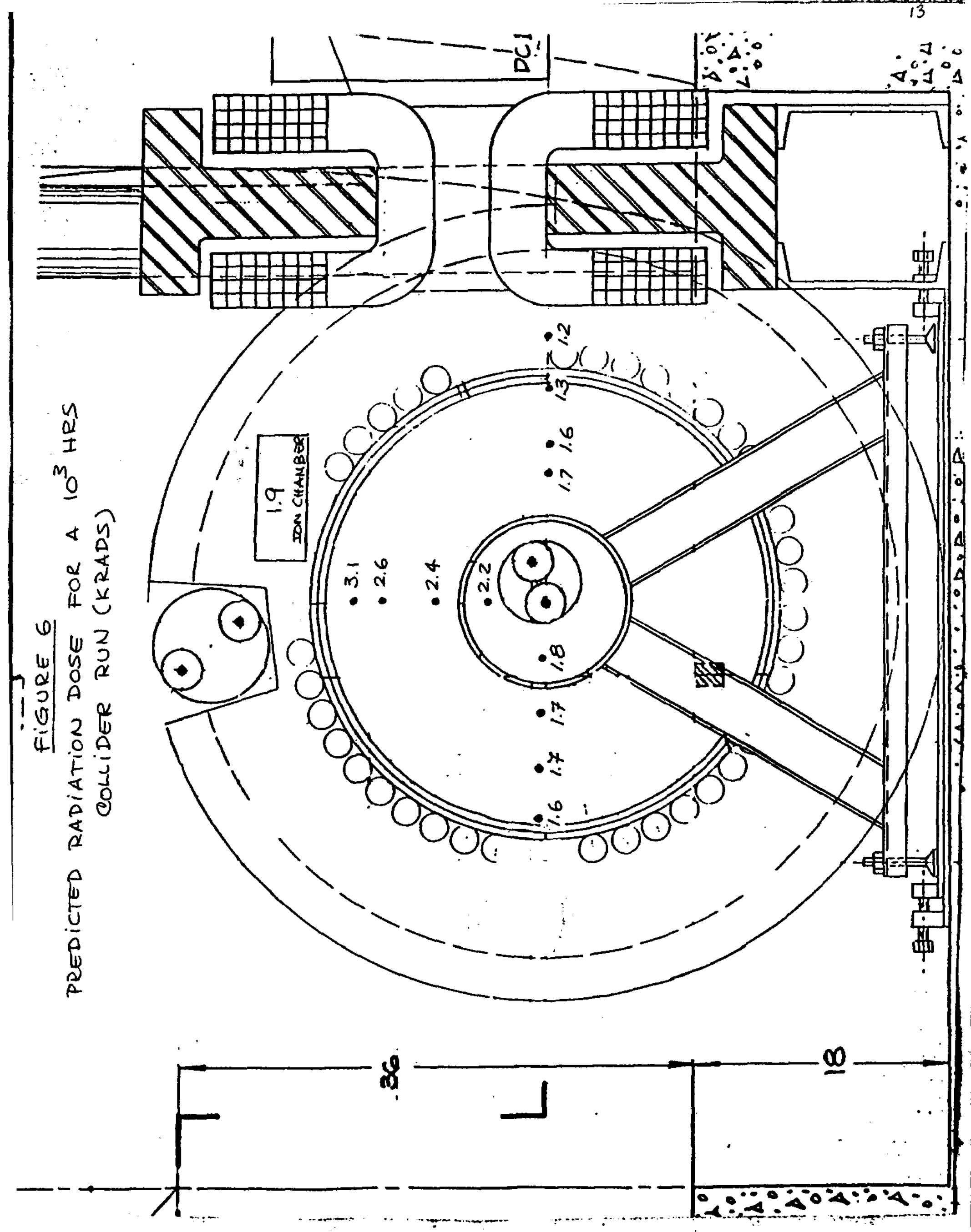

